\title{
Mapeamento de características de sites de compartilhamento de refeições baseado no Modelo 3 C de Colaboração
}

\author{
Francisco Gómez Castro \\ Berenice Santos Gonçalves \\ Doutor em Design. Universidade Federal de Santa Catarina (UFSC) - Brasil. francisco.gomez.castro1@gmail.com \\ Doutora em Engenharia de Produção. Universidade Federal de Santa Catarina (UFSC) - Brasil. berenice@cce.ufsc.br \\ Luiz Fernando Figueiredo \\ Doutor em Engenharia de Produção. Universidade Federal de Santa Catarina (UFSC) - Brasil. lff@cce.ufsc.br
}

\section{RESUMO}

O presente artigo tem como principal objetivo identificar as características e funcionalidades dos sites do tipo meal sharing - plataformas de compartilhamento de refeições, organizadas por profissionais ou por cozinheiros domésticos, nas quais os usuários buscam uma experiência gastronômica. Para tanto, apresentase um estudo e mapeamento das características dos referidos sites a partir da análise de domínio baseada na técnica Feature Oriented Domain Analysis (FODA) do modelo $3 C$ de colaboração, que organiza as atividades dos sistemas colaborativos nas dimensões de comunicação, coordenação e cooperação. Os resultados do levantamento contribuem para os negócios gastronômicos, evidenciando a possibilidade de sua implementação ou interpretação para a melhora do tipo de experiência gastronômica dirigida aos novos perfis de consumo. $\mathrm{O}$ modelo $3 \mathrm{C}$ de colaboração auxiliou no processo de organização das características utilizadas nas plataformas de compartilhamento de refeições. A partir da análise realizada, foi possível identificar e compreender a evolução das características e contextualizar as novas formas de socialização e interação entre as pessoas ao redor da gastronomia, considerando aquelas que possam ser trazidas aos sites de negócio gastronômico.

Palavras-chave: meal sharing; consumo colaborativo; sistemas colaborativos.

\section{Mapping features dining sharing sites based on Model 3C Collaboration}

\begin{abstract}
This article aims to identify the features of sites containing meal sharing platforms, organized by professionals or by domestic cooks, in which users are looking for a gastronomic experience. The article presents a study and mapping of the features of sites from the domain analysis based on the technical Feature Oriented Domain Analysis (FODA) of the $3 \mathrm{C}$ collaboration model. This model organizes the activities of collaborative systems in the dimensions of communication, coordination and cooperation. The survey results contribute to the gastronomy business, highlighting the possibility of their implementation or interpretation to improve the type of gastronomic experience that leads to new consumption patterns. The $3 \mathrm{C}$ collaborative model assisted in the organizing process of the characteristics used in the meal-sharing platforms. Based on the analysis performed, it was possible to identify and understand the evolution of the characteristics as well as to contextualize the new forms of socialization and interaction among people around gastronomy, considering those that can be brought to the gastronomy business sites.
\end{abstract}

Keywords: meal sharing; collaborative consumption; collaborative systems. 


\section{Introdução}

Os modelos de negócio baseados no consumo colaborativo dia a dia têm aumentado em setores e atividades econômicas onde a comunicação, coordenação e cooperação são feitas a partir de plataformas digitais ou redes sociais pela Internet. As pessoas vinculadas a uma atividade interagem para o planejamento e posterior desfrute da sua experiência. Essa realidade também envolve a atividade gastronômica. Por isso, o presente artigo tem como principal objetivo identificar as características e funcionalidades dos sites do tipo meal sharing.

Esses tipos de sites são plataformas de compartilhamento de refeições, organizadas por profissionais ou por cozinheiros domésticos, nas quais os usuários buscam uma experiência gastronômica. Para tanto, apresenta-se um estudo e mapeamento das características dos referidos sites a partir da análise de domínio baseada na técnica Feature Oriented Domain Analysis (FODA) do modelo $3 \mathrm{C}$ de colaboração que organiza as atividades dos sistemas colaborativos nas dimensões de comunicação, coordenação e cooperação para o estudo dos sistemas colaborativos.

\section{Referencial Teórico}

Esta seção apresenta uma revisão da literatura dos tópicos relevantes para este trabalho: (i) interação pessoa-computador, a interface e os sistemas colaborativos; (ii) o estilo de vida colaborativo e a experiência turística por meio dos sites de compartilhamento de refeições; (iii) os sistemas colaborativos; e (iv) o modelo 3C de colaboração na Web.

\section{Interação Pessoa-Computador, a Interface e os Sistemas Colaborativos}

A interação Pessoa-Computador $(\mathrm{HCl}$, Human-Computer Interaction) é uma área de estudo que foca na interação entre usuários e os sistemas informáticos, cujo objetivo é proporcionar as bases teóricas, metodológicas e práticas para o design e avaliação de produtos interativos que possam ser usados de forma eficiente, eficaz, segura e satisfatória. (Hassan, Martín; 2005). As variáveis que intervêm neste fenômeno interativo são muito diversas. Segundo Rogers, Sharp e Preece (2014), interação é interdisciplinar em sua prática e multidisciplinar em sua origem. Assim, as disciplinas envolvidas no design de interação, entre outras, são: a sociologia cognitiva e da conduta, a ergonomia, a antropologia, a sociologia e as ciências da computação. (Rozanski E Haake, 2003; Rogers, Sharp, Preece, 2014).

Por outro lado, a interface pode ser vista como uma linguagem de comunicação disponível para a interação usuário-sistema. IHC se refere à interação entre pessoas e sistemas computacionais, o que ocorre por meio da interface física ou de software. No caso de sistemas colaborativos, em que a interação entre usuários é feita por meio do sistema, a interface é também a linguagem para a interação entre usuários. Toda a interação do usuário com a funcionalidade do sistema é feita a partir da interface. O sucesso do sistema depende da qualidade de uso do sistema, ou seja, da qualidade da interação e da interface oferecida ao usuário (Oliveira 2010). A qualidade de uso de um sistema é definida por diferentes critérios. Cada critério foca em determinadas características da interface e da interação que as tornam adequadas para atingir os efeitos esperados ou desejados durante o uso. As qualidades de uso descritas, segundo Oliveira (2010), são: usabilidade, sociabilidade, comunicabilidade e acessibilidade.

Assim, pelo exposto, o presente artigo busca identificar as principiais características e funcionalidades das interfaces dos sites de compartilhamento de refeições (Meal sharing), destacando a funcionalidade e, sobretudo, as potencialidades de colaboração e comunicação. Segundo o site ConsumoColaborativo.cc", Meal Sharing é "a plataforma utilizada por sites espalhados por todo mundo para o compartilhamento de refeições. Através dos sites você encontra cozinheiros domésticos e chefs oferecendo refeições em suas casas, basta informar sua localização, escolher o menu que mais Ihe agrada e fazer sua reserva".

\footnotetext{
${ }^{1}$ Recuperado em 5 maio, 2015, de: http://consumocolaborativo.cc
} 


\section{O Estilo de Vida Colaborativo e a Experiência Turística por Meio dos Sites de Compartilhamento de Refeições}

Segundo Douglas e Isherwood (2013), considera-se que, por meio do consumo, são estruturadas relações sociais. Por meio do consumo, demonstram-se afetos e se demarcam lugares no mundo. Por isso, as relações de consumo são um meio de expressão de valores, desejos e de constituição dos sujeitos ${ }^{2}$. Rifkin (2000) afirma que vivemos na "era do acesso", momento no qual a utilização do bem, o que ele proporciona, é mais importante do que a propriedade sobre ele. Ideia similar apresenta Douglas (2007), que, desde a década de 1970, defende que as coisas não são necessárias por elas mesmas e sim pelas relações que as sustentam. Mas, Rifkin (2000) traz uma interessante observação: ressalta que atualmente estamos buscando as relações que nos importam ao consumir bens, como sempre, mas sem possuí-los, sem a necessidade de adquiri-los.

No chamado "consumo colaborativo", observam-se singulares formas de relacionamento entre os sujeitos: ao invés de comprar um produto novo, troca-se por outro. No lugar de pagar por uma propriedade, prefere-se alugar uma quando se precisa, ou, ao viajar, opta-se por ficar na casa de uma pessoa desconhecida para conhecer sua cultura e a cidade sob outra perspectiva, em detrimento de ficar em um hotel e usar uma agência de turismo.

Botsman e Rogers (2011) consideram que as práticas de consumo colaborativo podem ser organizadas em três sistemas distintos: "Serviços e Produtos", que consiste em pagar pelo benefício do objeto sem adquiri-lo para si; "Mercados de Redistribuição", que diz respeito às trocas que aumentam o ciclo de vida dos produtos; e "Estilos de Vida Colaborativos", que tem relação com partilhas imateriais. Este estudo está focado nos "Estilos de Vida Colaborativos", em particular na experiência do compartilhamento de refeições.

Um exemplo dessa experiência é o EatWith, fundado em 2012 em Tel Aviv/Israel e hoje com anfitriões em 17 cidades no mundo, que permite agendar uma refeição com habitantes locais pagando um preço preestabelecido. Os anfitriões podem se cadastrar no site e oferecer refeições em sua casa para pessoas (em geral turistas) "em busca de uma comida local sem a impessoalidade de um restaurante, uma boa conversa ou conhecer a cultura". Em cada perfil ficam registradas as comidas que podem ser preparadas, informações pessoais dos anfitriões, preços por pessoa, comentários de quem já experimentou e fotos. Outra iniciativa parecida é o Meal Sharing, com usuários em mais de 400 cidades no mundo, que também reúne as pessoas em torno da mesa de refeição, mas sem pagar.

Sharing economy (economia de compartilhamento) é como se chama o mercado crescente de serviços que se baseiam em trocas, empréstimo ou aluguel de itens pessoais para locais ou viajantes. A economia de compartilhamento, em relação ao setor turístico, está gerando um importante número de microempreendedores e um crescimento da economia informal (Ramos; Gómez, 2006). Algumas informações citam, inclusive, montantes muito elevados em nível estadual na Europa (Merino, 2014).

Nesse contexto, surgiram novos modelos de negócio no setor turístico com implicações no cenário P2P33. Segundo Romero (2014), as novas tendências turísticas do usuário estão baseadas nas experiências locais, em serviços complementares, no valor humano e na ubiquação. Desses novos comportamentos no lazer turístico baseados em "estilos de vida colaborativos" surge o conceito de Meal Sharing, uma forma de conhecer a gastronomia local por anfitriões nativos do lugar que o turista visita. Essa modalidade está tendo sucesso na Europa, sobretudo na Alemanha, e nos Estados Unidos. Alguns dos sites mais conhecidos são: Meal Sharing ${ }^{4}$, Eat With ${ }^{5}$, EatFeasty ${ }^{6}$, Eat

\footnotetext{
2 Sujeito tal como é entendido por Foucault (2005).

${ }^{3}$ Segundo Michel Bauwens, em "The Political Economy of Peer Production" ("A Economia Política da Produção entre Pares"), à medida que os sistemas sociais, econômicos e políticos se transformam em redes distribuídas, surge o modelo peer to peer (P2P), ponto a ponto, como uma nova dinâmica produtiva. Mais que uma nova tecnologia de comunicação, é o modelo de funcionamento de novos processos sociais e faz surgir um terceiro modo de produção, de autoridade e de propriedade, e visa aumentar a participação generalizada de atores equipotenciais.

The Political Economy of Peer Production, Recuperado em 25 maio, 2015, de: http://www.ctheory.net/articles.aspx?id=499 e em www.p2pfoundation.net/

${ }^{4}$ www.mealsharing.com
} 
with a local ${ }^{7}$ Cookening ${ }^{8}$. Alguns são uma plataforma em que anfitriões oferecem uma refeição caseira por um preço preestabelecido, outros servem para unir pessoas em torno de uma mesa sem pagar.

\section{Os Sistemas Colaborativos}

Sistemas Colaborativos é a tradução adotada no Brasil para designar os termos groupwere e CSCW (Computer Supported Cooperative Work). Muitos os consideram sinônimos, outros reservam a palavra groupwere para designar especificamente os sistemas computacionais usados para apoiar o trabalho em grupo e o termo CSCW (Computer Supported Cooperative Work) para designar tanto os sistemas quanto os efeitos psicológicos, sociais e organizacionais do uso desses sistemas. A área surgiu no inicio da década de 1980, através de um esforço dos tecnólogos em aprender com a Psicologia, a Sociologia, a Antropologia, a Educação, a Economia e outras áreas que investigam a atividade em grupo. Na área de Sistemas Colaborativos, discute-se tanto o uso quanto o desenvolvimento de sistemas para dar suporte à colaboração. Diversas áreas da computação contribuem para os aspectos técnicos relacionados ao desenvolvimento de sistemas colaborativos: Sistemas de Informação, Interação Humano-Computador, Inteligência Artificial, Sistemas Distribuídos, Banco de Dados, Computação Gráfica, Sistemas Multimídia, Engenharia de Software, entre outras (Fuks, 2012).

Sistemas Colaborativos são ferramentas computacionais utilizadas em redes de comunicação para facilitar a execução de trabalhos em grupo. Essas ferramentas devem oferecer aos seus usuários formas de interação, facilitando o controle, a coordenação, a colaboração e a comunicação entre as partes que compõem o grupo, tanto no mesmo local, como em locais diferentes, as formas de interação acontecendo ou não ao mesmo tempo. Percebe-se, com isso, que os Sistemas Colaborativos possuem o objetivo de diminuir as barreiras impostas pelo espaço físico e pelo tempo. (Camargo, Khouri \& Giarola, 2005).

As teorias e modelos da área de Sistemas Colaborativos nos auxiliam a selecionar e projetar sistemas para dar suporte ao trabalho em grupo (CS - Computer Supported), a partir da compreensão de como as pessoas colaboram, incluindo os aspectos sociais relacionados ao uso da tecnologia (CW-Cooperative Wok). As referidas teorias são úteis para entender, comparar, abstrair e generalizar as observações sobre o mundo que nos cerca e sobre os produtos criados na sociedade.

\section{O Modelo 3C de Colaboração na Web}

De acordo com Ellis et al. (1991), a colaboração é vista como uma combinação de comunicação, coordenação e cooperação. Essa maneira de analisar a colaboração é conhecida na literatura como modelo $3 \mathrm{C}$ e seu diagrama pode ser visto na Figura 1. O Modelo $3 \mathrm{C}$ de Colaboração (Figura 1) analisa a colaboração em três dimensões: comunicação, coordenação e cooperação. A comunicação é caracterizada pela troca de mensagens, pela argumentação e pela negociação entre pessoas; a coordenação é caracterizada pelo gerenciamento de pessoas, atividades e recursos, e a cooperação é caracterizada pela atuação conjunta no espaço compartilhado para a produção de objetos ou informações (Fuks, 2012).

\footnotetext{
${ }^{5}$ www.eatwith.com

${ }^{6}$ www.eatfeastly.com

http://eatwithalocal.socialgo.com

${ }^{8}$ www.cookening.com
} 


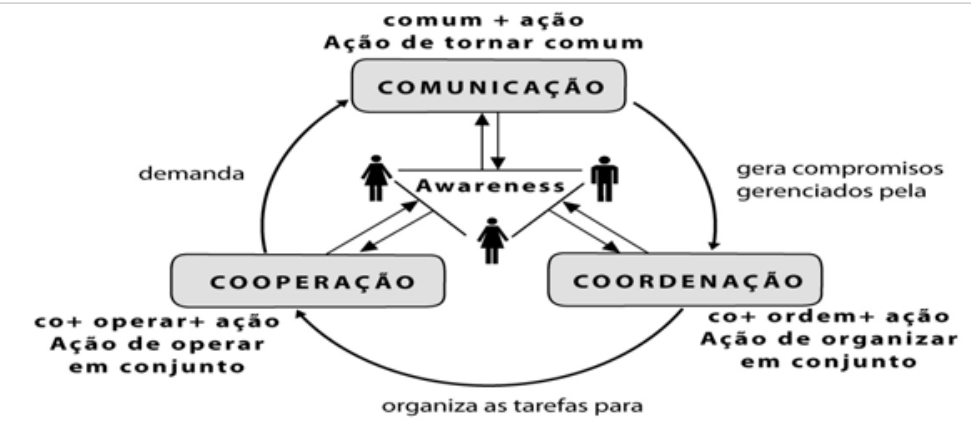

Figura 1. Diagrama do modelo $3 \mathrm{C}$

Fonte: Gerosa (2006)

O modelo $3 \mathrm{C}$ aparece frequentemente na literatura como um meio de classificar sistemas colaborativos $^{9}{ }^{10}$. Um sistema colaborativo geralmente integra um conjunto de ferramentas para colaboração. Segundo Pimentel e Gerosa $(2006)^{11}$, é possível identificar os elementos constituintes de uma família de aplicação classificando-os em função do modelo 3C, tal como apresentado no Quadro 1, para o domínio das ferramentas de comunicação síncrona e para o estudo de sistemas colaborativos baseado no modelo $3 \mathrm{C}$ de Colaboração.

\section{Quadro 1. Classificação 3C dos elementos das ferramentas de comunicação síncrona}

\begin{tabular}{|c|c|c|}
\hline \multirow{5}{*}{ Comunicação } & Linguagem & $\begin{array}{l}\text { Principais linguagens da comunicação humana: escrita } \\
\text { (texto), falada (áudio), pictórica (imagens e animações) e } \\
\text { gestual (vídeo e avatar). }\end{array}$ \\
\hline & Transmissão & $\begin{array}{l}\text { Pontual (após o emissor formular toda a mensagem) ou } \\
\text { Contínua (transmissão contínua de vídeo e áudio, ou } \\
\text { caractere-a-caractere enquanto a mensagem está sendo } \\
\text { formulada) }\end{array}$ \\
\hline & $\begin{array}{l}\text { Tamanho e } \\
\text { Qualidade }\end{array}$ & $\begin{array}{l}\text { Tamanho: quantidade de caracteres (texto) ou a duração em } \\
\text { segundos (vídeo e áudio). Qualidade do vídeo e do áudio é } \\
\text { geralmente reduzida para a transmissão pela Internet }\end{array}$ \\
\hline & $\begin{array}{l}\text { Estruturação do } \\
\text { discurso }\end{array}$ & $\begin{array}{l}\text { Estrutura linear (uma mensagem apresentada após a outra), } \\
\text { hierárquica (árvore, threads) ou em rede (gráficos, mapas) }\end{array}$ \\
\hline & Categorização & $\begin{array}{l}\text { Rótulos para caracterizar as mensagens, tais como: tipo de } \\
\text { fala (sussurra, pergunta, concorda etc.); tipo de discurso } \\
\text { (direto ou indireto); tipo de emoção (alegre, zangado etc.) }\end{array}$ \\
\hline \multirow{9}{*}{ Coordenação } & Tópico & Assunto a ser discutido \\
\hline & Sessão & Espaço de tempo para a duração da conversação \\
\hline & Acesso & Quem ou quantos podem participar da conversação \\
\hline & Presença & Quem está participando da conversação \\
\hline & Disponibilidade & Status do participante: presente, ausente, ocupado etc. \\
\hline & Papéis & Atribuição de papéis: Operador, Mediador, Moderador etc. \\
\hline & Posse da palavra & Quem pode falar em um dado momento \\
\hline & Frequência & $\begin{array}{l}\text { Limite da quantidade de mensagens que podem ser } \\
\text { enviadas num intervalo de tempo }\end{array}$ \\
\hline & Visibilidade & $\begin{array}{l}\text { Pública (visível para todos os participantes) ou particular } \\
\text { (restrita a dois participantes) }\end{array}$ \\
\hline
\end{tabular}

\footnotetext{
${ }^{9}$ Borges R. M., Pinto S. C. C. S., Barbosa J. L. V., Barbosa D. N. F. (2007) Usando o modelo 3C de colaboração e Vygotsky no ensino de programação distribuída em pares. Workshop em Informática na Educação (sbie) 2007 XVIII Simpósio Brasileiro de Informática na Educação - SBIE.

${ }^{10}$ Borghoff U. M., Schlichter J. H. (1996) Computer-supported cooperative work: introduction to distributed applications

${ }^{11}$ Pimentel, M., Gerosa, M.A., Filippo, D., Raposo, A., Fuks, H. \& Lucena, C.J.P. (2006) "Modelo 3 C de Colaboração no Desenvolvimento de Sistemas Colaborativos", Anais do III Simpósio Brasileiro de Sistemas Colaborativos, Natal - RN, 20 a 22 de Novembro de 2006. ISBN 857669-097-7. Porto Alegre: SBSC, 2006. pp. 58-67
} 


\begin{tabular}{|l|l|l|}
\hline \multirow{1}{*}{ Cooperação } & Endereçamento & Indicação do destinatário da mensagem \\
\cline { 2 - 3 } & $\begin{array}{l}\text { Indicação do } \\
\text { turno em } \\
\text { desenvolvimento }\end{array}$ & $\begin{array}{l}\text { Informação de que o participante está formulando a } \\
\text { mensagem (antes de sua transmissão pontual) }\end{array}$ \\
\hline Avaliação & $\begin{array}{l}\text { Qualificação das mensagens, dos participantes ou da sessão } \\
\text { espaço }\end{array}$ & Armazenamento das mensagens publicadas \\
\cline { 2 - 4 } & $\begin{array}{l}\text { Rensagens } \\
\text { preconcebidas }\end{array}$ & $\begin{array}{l}\text { Mensagens pré-elaboradas disponíveis para os participantes } \\
\text { trocarem durante a conversação }\end{array}$ \\
\hline
\end{tabular}

Fonte: Pimentel e Gerosa (2006).

\section{Procedimentos Metodológicos}

\section{Classificação da Pesquisa}

Esta pesquisa, em relação às formas de classificação, é básica, segundo Gil (2002). Do ponto de vista da sua natureza e da forma de abordagem do problema, é qualitativa. Também pode ser considerada exploratória do ponto de vista de seus objetivos. Com relação aos procedimentos técnicos, pode ser considerada descritiva.

\section{A Construção da Amostra dos Sites de Estudo}

Em relação ao tipo de amostra, referente aos sites escolhidos para esse estudo, segundo Fragoso, Recuero e Amaral (2011), foi intencional, pois procurou-se amostras representativas cujos elementos são selecionados conforme critérios que derivam do problema da pesquisa, das características do universo observado e das condições e métodos de observação e análise.

Segundo os subtipos de amostras intencionais que propõem as autoras, a amostra do estudo pode ser caracterizada pelos seguintes subtipos: por intensidade e por critério. Por intensidade, posto que a seleção de sites favorece os elementos em que as características que interessam à pesquisa estão presentes de forma intensa ou evidente, mas que não se caracterizam como casos extremos. Localizando a observação em casos ou elementos nos quais a informação é mais densa ou fácil de verificar e colocando em foco características previamente definidas. Por outro lado, a caracterização por critério, refere-se ao fato de que foram selecionados os sites que apresentavam uma determinada característica ou critério predefinido.

\section{Etapas da Pesquisa}

O estudo foi conduzido a partir das quatro principais etapas citadas e detalhas a seguir:

1. Escolha dos sites;

2. Características levantadas pelos autores e que serão consideradas na análise;

3. Resultados: análise propriamente dita;

4. Conclusões.

\section{Etapa 1. Escolha dos sites.}

Considerando as classificações da amostra expostas anteriormente, entre os sites selecionados para representar itens que permitem o compartilhamento de refeições online, foram levantadas as características do domínio. Para executar esse levantamento, foi feita uma análise exploratória das características disponibilizadas pelos sites avaliados e uma filtragem de quais dessas características possibilitam a exploração das funcionalidades pelas que os usuários 
realizaram a comunicação que terminava com o desfrute da uma experiência ligada aos estilos de vida colaborativos e à atividade particular do compartilhamento de refeições. Nessa primeira etapa, foram escolhidos sites segundo três critérios: representatividade do conceito de estudo (Meal sharing) ${ }^{12}$, avaliação da fonte e popularidade. Assim, foram avaliados os dez sites do Quadro 2.

\section{Quadro 2. Lista de sites avaliados}

\begin{tabular}{|c|l|l|l|}
\hline \multicolumn{3}{|c|}{ Lista de sites avaliados } \\
\hline 10 & www.mealsharing.com & $6^{\circ}$ & www.grubclub.com \\
\hline $2^{\circ}$ & www.eatwith.com/list/brazil/ & 70 & www.kitchensurfing.com \\
\hline $3^{\circ}$ & www.shareyourmeal.net & $8^{\circ}$ & www.dinneer.com \\
\hline $4^{\circ}$ & eatwithalocal.socialgo.com & $9^{\circ}$ & www.bonappetour.com \\
\hline $5^{\circ}$ & eatfeastly.com & $10^{\circ}$ & www.bookalokal.com \\
\hline
\end{tabular}

Etapa 2. Características levantadas pelos autores e que serão consideradas na análise.

O levantamento das características (Quadro 3), assim como as definições de cada uma das características (Quadro 4) que foram consideradas para o presente estudo foram extraidas dos trabalhos dos pesquisadores: Oliveira e Gerosa (2010), Michalsky, Mamani e Gerosa (2010), e Michalsky e Gerosa (2011). Segundo os autores, as características do domínio proposto foram descritas utilizando-se o padrão proposto por Schummer \& Lukosch ${ }^{13}$ com algumas simplificações na descrição de padrões de interação mediada por computador baseada nas características mapeadas nas redes sociais ${ }^{14}$.

\section{Etapa 3. Resultados: Análise propriamente dita.}

O conjunto de características selecionadas para o mapeamento de funções dos sites de compartilhamento de refeições foi classificado segundo o modelo $3 C$ de colaboração. Essa avaliação das características dos sites foi feita visualmente, através de imagens capturadas de cada site avaliado, que, depois, foram classificadas de acordo com sua função no modelo 3C. Foi usada a técnica Feature Oriented Domain Analysis (FODA) para a análise de domínio, pois era preciso identificar, coletar e organizar informações relevantes do domínio, utilizando o conhecimento existente e técnicas para modelagem de informação ${ }^{15}$ da Engenharia de Domínio. Os resultados obtidos estão expostos no quadro 5.

\section{Etapa 4. Conclusões.}

Na última parte deste artigo poderão ser lidas as ligações entre as problemáticas e objetivos observados no estudo, onde são propostas novas linhas de pesquisa e considerações para os negócios relacionados à atividade gastronômica.

\section{Materiais e Métodos: Análise de Domínio, Técnica Feature Oriented Domain Analysis (FODA)}

\footnotetext{
12 Segundo o site Consumo Colaborativo: "Meal Sharing, essa é a plataforma utilizada por sites espalhados por todo mundo para o compartilhamento de refeições. Através dos sites você encontra cozinheiros domésticos e chefs oferencendo refeições em suas casas, basta informar sua localização, escolher o menu que mais lhe agrada e fazer sua reserva". Recuperado em 25 maio, 2015, de: http://consumocolaborativo.cc

13 Schummer, T.; Lukosch, S. (2007) Patterns for Computer-Mediated Interaction. West Sussex: John Wiley \& Sons Ltd.

${ }^{14}$ Oliveira, L. S.; Gerosa, Marco A. "Uma Engenharia de Domínio Baseada em Padrões de Interação e no Modelo $3 C$ de Colaboração para Redes Sociais de Compartilhamento na Web 2.0". WEBMEDIA 2010, Simpósio Brasileiro de Sistemas Multimídia e Web, Volume II

${ }^{15}$ Kang, K. C. et al. (1990) Feature-Oriented Domain Analysis (FODA) Feasibility Study. : CMU/SEI.
} 
A análise de domínio é uma das etapas da Engenharia de Domínio. De acordo com Prieto \& Rango $(1991)^{16}$, a Engenharia de Domínio possibilita que características comuns e variáveis sejam identificadas e organizadas utilizando um processo previamente definido. Ela busca identificar, coletar e organizar informações relevantes do domínio, utilizando para tal o conhecimento existente e técnicas para modelagem de informação ${ }^{17}$. Neste trabalho, será utilizada a técnica Feature Oriented Domain Analysis - FODA. Assim, as informações obtidas na análise possibilitam determinar os limites que norteiam o domínio, as características comuns e as variáveis de aplicações do domínio. Além de gerar, como resultados, modelos para a representação de tais características. A modelagem FODA é orientada a características (features). De acordo com Kang et al. $(1990)^{18}$, essas características são atributos do sistema que afetam diretamente os usuários finais.

\section{Análise e Discussão de Resultados}

A análise se pautou no domínio de dez sites que permitem o compartilhamento de refeições. Eles foram escolhidos por três critérios: representatividade do conceito de estudo ${ }^{19}$, avaliação da fonte e popularidade, como dito anteriormente. A representatividade do conceito de estudo refere-se àqueles sites baseados em estilos de vida colaborativos que realizam o compartilhamento de refeições em modelos P2P. Em relação à fonte, foram escolhidos aqueles sites publicados nos portais ColaborativeConsuption. $\mathrm{Com}^{20}$ e ConsumoColaborativo.cc ${ }^{21}$. E a respeito da popularidade, foram usadas as informações da empresa Alexa ${ }^{22}$, ordenando por popularidade os sites (Quadro 2).

Entre os sites selecionados para representar itens que permitem o compartilhamento de refeições online, foram levantadas as características do domínio. Para executar esse levantamento, foi realizada uma análise exploratória das características disponibilizadas pelos sites avaliados e uma filtragem de quais dessas características possibilitam a exploração das funcionalidades pelas quais os usuários realizaram a comunicação que terminava com o desfrute da uma experiência ligada aos estilos de vida colaborativos e à atividade particular do compartilhamento de refeições.

O objetivo do levantamento e mapeamento dessas características é conhecer os canais, agentes e formas categorizadas pelo modelo $3 C$ de colaboração. $E$, também, conseguir uma perspectiva o mais abrangente possível das novas formas de lazer, experiências de consumo, interação entre pessoas e novos modelos de negocio P2P a partir das estruturas implementadas nos sites, que, afinal, são as que possibilitam as diferentes formas de comunicação, coordenação e cooperação. O levantamento dessas características (Quadro 3) foi executado segundo os trabalhos dos pesquisadores: Oliveira e Gerosa (2010), Michalsky, Mamani e Gerosa (2010), e Michalsky e Gerosa (2011). Segundo os autores, essas características do domínio proposto foram descritas utilizando-se o padrão proposto por Schummer \& Lukosch $^{23} \mathrm{com}$ algumas simplificações na descrição de padrões de interação mediada por computador baseada nas características mapeadas nas redes sociais ${ }^{24}$. A comparação mostra a lista de características levantadas dos sites de jornalismo online ${ }^{25}$ e as características das possíveis fontes de dados ${ }^{26}$ (quadro 3). A avaliação das

\footnotetext{
${ }^{16}$ Prieto-Diaz, R.; Arango, G. (1991) Domain Analysis Concepts and Research Directions". In: Prieto-Diaz, R., Arango, G. (eds), Domain Analysis and Software Systems Modeling. : IEEE Computer Society Press.

17 Kang, K. C. et al. (1990) Feature-Oriented Domain Analysis (FODA) Feasibility Study. : CMU/SEI.

18 Kang, K. C. et al. (1990) Feature-Oriented Domain Analysis (FODA) Feasibility Study. : CMU/SEI.

19 Segundo o site de consumo colaborativo Brasil "Meal Sharing, essa é a plataforma utilizada por sites espalhados por todo mundo para o compartilhamento de refeições. Através dos sites você encontra cozinheiros domésticos e chefs oferencendo refeições em suas casas, basta informar sua localização, escolher o menu que mais lhe agrada e fazer sua reserva." Recuperado em 27 maio, 2015 , de: http://www.consumocolaborativo.cc/meal-sharing-refeicoes-caseiras-ao-redor-mundo/

20 Recuperado em 27 maio, 2015, de:

http://www.consumocolaborativo.cc/meal-sharing-refeicoes-caseiras-ao-redor-mundo/

${ }^{21}$ Recuperado em 13 maio, 2015, de: http://www.collaborativeconsumption.com/directory/?cats=93

22 Recuperado em 29 maio, 2015, de: http://www.alexa.com

${ }^{23}$ Schummer, T.; Lukosch, S. (2007) Patterns for Computer-Mediated Interaction. West Sussex: John Wiley \& Sons Ltd.

${ }^{24}$ Oliveira, L. S.; Gerosa, Marco A. "Uma Engenharia de Domínio Baseada em Padrões de Interação e no Modelo $3 C$ de Colaboração para

Redes Sociais de Compartilhamento na Web 2.0". WEBMEDIA 2010, Simpósio Brasileiro de Sistemas Multimídia e Web, Volume II

${ }^{25}$ Michalsky, S.; Mamani, E. Z. S.; Gerosa, Marco A. "A inteligência coletiva na Web: Uma análise de domínio para o jornalismo online". WebMedia 2010 - Simpósio Brasileiro de Sistemas Multimídia e Web
} 
características foi feita visualmente, através de imagens capturadas de cada site avaliado, que foram classificadas de acordo com sua função no modelo $3 C$.

\section{Quadro 3. Comparação e Seleção de Características mapeadas nas redes sociais e sites em estudos baseados no modelo $3 \mathrm{C}$ de colaboração}

\begin{tabular}{|c|c|c|c|c|}
\hline \multicolumn{5}{|c|}{ Características mapeadas em redes sociais e sites. } \\
\hline & \multirow[b]{2}{*}{$\begin{array}{l}\text { Características } \\
\text { mapeadas nas redes } \\
\text { sociais (Oliveira, Gerosa } \\
\& \text { Marco, 2010) }{ }^{27}\end{array}$} & \multirow[b]{2}{*}{$\begin{array}{l}\text { Características } \\
\text { levantadas nos sites de } \\
\text { jornalismo online } \\
\text { (Michalsky, Mamani, } \\
\text { Gerosa \& Marco; 2010) } \\
28\end{array}$} & \multirow[b]{2}{*}{$\begin{array}{l}\text { Características das } \\
\text { possíveis fontes de dados } \\
\text { (Michalsky, Gerosa \& } \\
\text { Marco; 2011) }{ }^{29}\end{array}$} & Seleção \\
\hline & & & & $\begin{array}{l}\text { Conjunto de características para } 0 \\
\text { mapeamento de funções dos sites de } \\
\text { compartilhamento de refeições }\end{array}$ \\
\hline \multirow{3}{*}{ Comunicação } & Comentário & Comentário & Comentário & Comentário \\
\hline & & Enquete & Enquete & Enquete \\
\hline & & Lista de Discussão & Lista de Discussão & Lista de Discussão \\
\hline \multirow{5}{*}{ Coordenação } & & Seguir tópico & Seguir tópico & Seguir tópico \\
\hline & Atividades recentes & & & \\
\hline & Buscar pessoas & & & \\
\hline & Grupos & & & \\
\hline & Denunciar & Denúncia & Denúncia & Denúncia \\
\hline \multirow{18}{*}{ Cooperação } & Compartilhar objetos & Compartilhamento & Compartilhamento & Compartilhamento \\
\hline & Avaliação & Avaliação & Avaliação & Avaliação \\
\hline & & Produção & Produção & Produção \\
\hline & & Indicação & Indicação & Indicação \\
\hline & & Upload & Upload & Upload \\
\hline & & Busca & Busca & Busca \\
\hline & Tags & Tags & Tags & Tags \\
\hline & Estatística & & & \\
\hline & Exportar & & & \\
\hline & Descrição & & & \\
\hline & Recomendação & & & \\
\hline & Subir & & & \\
\hline & Marcar & & & \\
\hline & Categorias & & & \\
\hline & Buscar objetos & & & \\
\hline & Promoção & & & \\
\hline & Playlist ou Álbum & & & \\
\hline & Favoritos & & Favoritos & Favoritos \\
\hline
\end{tabular}

\footnotetext{
${ }^{26}$ Michalsky, S.; Gerosa, Marco A. "Um modelo para o suporte computacional da inteligência coletiva na Web". SBSC 2011 - VIII Simpósio Brasileiro de Sistemas Colaborativo

${ }^{27}$ Oliveira, L. S.; Gerosa, Marco A. "Uma Engenharia de Domínio Baseada em Padrões de Interação e no Modelo 3 C de Colaboração para Redes Sociais de Compartilhamento na Web 2.0". WEBMEDIA 2010, Simpósio Brasileiro de Sistemas Multimídia e Web, Volume II

${ }^{28}$ Michalsky, S.; Mamani, E. Z. S.; Gerosa, Marco A. "A inteligência coletiva na Web: Uma análise de domínio para o jornalismo online". WebMedia 2010 - Simpósio Brasileiro de Sistemas Multimídia e Web

${ }^{29}$ Michalsky, S.; Gerosa, Marco A. "Um modelo para o suporte computacional da inteligência coletiva na Web". SBSC 2011 - VIII Simpósio Brasileiro de Sistemas Colaborativo
} 


\begin{tabular}{|l|l|l|l|l|}
\hline \multirow{4}{*}{} & Anotação & & & \\
\cline { 2 - 5 } & Permissão & & & \\
\cline { 2 - 5 } & & & Publicar status & Publicar status \\
\cline { 2 - 5 } & & Versão Impressão & Versão Impressão & \\
\cline { 2 - 5 } Individual & Salvar & Salvar & \\
\cline { 2 - 5 } & & & Login & Login \\
\cline { 2 - 5 } & & & Dados & Páses \\
\cline { 2 - 5 } & & & Localização & Localização \\
\hline
\end{tabular}

Fonte: Elaborado pelos autores

\section{Características Identificadas}

No quadro 4, apresenta-se cada característica a considerar na avaliação dos sites, segundo os estudos feitos por Oliveira, Gerosa \& Marco (2010), Michalsky, Mamani \&.Gerosa (2010), Michalsky, Gerosa \& Marco (2011).

Quadro 4. Lista de características levantadas baseada nos sites de jornalismo e adaptadas aos sites de compartilhamento de refeições

\begin{tabular}{|l|l|}
\hline Comentário & Possibilita aos usuários comentar sua experiência e opinião sobre propostas do anfitrião. \\
\hline Enquete & Possibilita aos usuários responder perguntas escolhendo uma dentre algumas alternativas predefinidas. \\
\hline Lista de Discussão & Possibilita a um grupo de usuários a troca de mensagens via e-mail entre todos os membros do grupo. \\
\hline Denúncia & Possibilita aos usuários notificar quando encontrarem algum conteúdo impróprio em objetos do sistema. \\
\hline Seguir tópico & $\begin{array}{l}\text { Possibilita aos usuários receber notificações quando exista alguma atualização de um objeto ou categoria do } \\
\text { sistema }\end{array}$ \\
\hline Compartilhamento & Possibilita aos usuários compartilhar a informação de um objeto do sistema com outros sistemas \\
\hline Avaliação & Possibilita aos usuários avaliar objetos do sistema. \\
\hline Produção & Possibilita aos usuários adicionar novas notícias no sistema. \\
\hline Indicação & Possibilita aos usuários indicar o conteúdo de um objeto do sistema \\
\hline Upload & Possibilita aos usuários adicionar arquivos tipo mídia (vídeo, imagens) em objetos do sistema. \\
\hline Busca & Possibilita aos usuários buscar informação no conteúdo dos objetos do sistema. \\
\hline Tags & Possibilita aos usuários visualizar e/ou caracterizar com palavras-chaves objetos do sistema. \\
\hline Login & Possibilita aos usuários acesso a seu cadastro e ao sistema interno \\
\hline Dados & Possibilita aos usuários salvar os dados de seu perfil para próximos acessos ao sistema. \\
\hline Localização & Possibilita aos usuários adicionar o lugar onde estão. \\
\hline
\end{tabular}

Fonte: Oliveira, Gerosa \& Marco (2010), Michalsky, Mamani \&. Gerosa (2010), Michalsky,

Gerosa \& Marco (2011) 


\section{Resultados}

Após a seleção e definição das características levantadas, a seguir, apresenta-se os resultados da análise dos sites de Meal Sharing, através do quadro 5:

Quadro 5. Resultados da análise dos sites de Meal Sharing

\begin{tabular}{|c|c|c|c|c|c|c|c|c|c|c|c|c|c|c|c|}
\hline \multirow[b]{2}{*}{ Características } & \multicolumn{3}{|c|}{ Comunicação } & \multicolumn{2}{|c|}{ Coordenação } & \multicolumn{7}{|c|}{ Cooperação } & \multicolumn{3}{|c|}{$\begin{array}{l}\text { Interação } \\
\text { Individual }\end{array}$} \\
\hline & 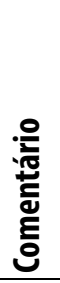 & 苂 & 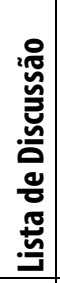 & 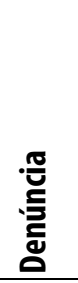 & 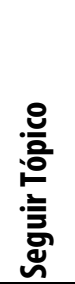 & 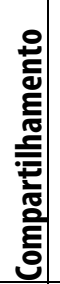 & 荵 & 胥 & 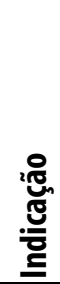 & $\frac{8}{5}$ & ङ & $\tilde{s}$ & కิ & $\begin{array}{l}\tilde{c} \\
\bar{\Xi} \\
\bar{a}\end{array}$ & 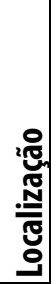 \\
\hline mealsharing.com & $\cdot$ & & & $\cdot$ & & $\cdot$ & $\cdot$ & & $\cdot$ & $\cdot$ & $\cdot$ & & $\cdot$ & $\cdot$ & - \\
\hline eatwith.com/list/brazi & $\cdot$ & & & - & & - & $\cdot$ & $\cdot$ & $\cdot$ & $\cdot$ & $\cdot$ & & - & - & - \\
\hline shareyourmeal.net & $\cdot$ & & & - & - & - & $\cdot$ & $\cdot$ & $\cdot$ & $\cdot$ & $\cdot$ & & - & - & - \\
\hline eatwithalocal.socialgo.com & & & & & & & & $\cdot$ & & $\cdot$ & & & - & - & $\cdot$ \\
\hline eatfeastly.com & $\cdot$ & & & $\cdot$ & $\cdot$ & $\cdot$ & $\cdot$ & $\cdot$ & $\cdot$ & $\cdot$ & $\cdot$ & & $\cdot$ & $\cdot$ & $\cdot$ \\
\hline grubclub.com & $\cdot$ & & & $\cdot$ & - & - & $\cdot$ & $\cdot$ & $\cdot$ & $\cdot$ & $\cdot$ & & • & • & $\cdot$ \\
\hline kitchensurfing.com & & & & $\cdot$ & & & $\cdot$ & & & & $\cdot$ & & $\cdot$ & $\cdot$ & $\cdot$ \\
\hline www.dinneer.com & $\cdot$ & & & $\cdot$ & $\cdot$ & $\cdot$ & $\cdot$ & $\cdot$ & $\cdot$ & $\cdot$ & $\cdot$ & & $\cdot$ & $\cdot$ & $\cdot$ \\
\hline bonappetour.com & & & & • & & • & $\cdot$ & & & $\cdot$ & $\cdot$ & & • & • & $\cdot$ \\
\hline bookalokal.com & • & & & • & • & • & • & . & • & • & • & & • & • & - \\
\hline
\end{tabular}

Fonte: Elaborado pelo autor

\section{Discussão de Resultados}

Segundo os resultados dos sites avaliados, as características enquete, lista de discussão e tags dos sites estudados não estão sendo usadas nesta tipologia de sites.

Por outro lado, as características login, dados e localização, em todos os sites, com exceção do Eatwithaloca ${ }^{\beta 0}$, são simplificadas para o usuário pela possibilidade da sua identificação a partir de seu perfil na rede social Facebook.

Finalmente, as demais características aqui consideradas terão um maior número de funcionalidades dependendo do foco do site. Ou seja, se o site focar na experiência gastronômica com moradores locais e cozinheiros domésticos, os números de funcionalidades das características dos perfis de usuário serão muito parecidos, até porque os convidados podem se tornar anfitriões. Porém, se o site está focado no compartilhamento de refeições feitas por um cozinheiro profissional que atua como anfitrião, as características terão maior número de funcionalidades no perfil desse último. Daí, tem-se que, neste último caso, o valor e os produtos sejam as informações mais frequentes.

Segundo os resultados dos sites avaliados, podemos ver como os sites oferecem características antes apenas consideradas em redes sociais, como login, dados ou localização,

\footnotetext{
${ }^{30}$ Recuperado em 30 maio, 2015, de: eatwithalocal.socialgo.com
} 
embora todos os sites tenham links e perfis nas redes sociais mais usadas atualmente. Também há um agrupamento de certas características, dependendo de se os sites são de cozinheiros profissionais, onde o perfil está mais desenvolvido, ou de cozinheiros domésticos, onde o importante é a interação entre pessoas.

\section{Conclusões}

O método $3 \mathrm{C}$ de colaboração, junto com as características levantadas na análise de domínio a partir da técnica FODA, permitem organizar aquelas características que estão sendo usadas pelos usuários nas plataformas de compartilhamento de refeições. Os conceitos de comunicação, coordenação e cooperação não são recentes, remontam aos anos 80 , como visto na parte inicial deste artigo. A evolução da socialização digital e dos sistemas colaborativos por meio da conectividade oferecida pela Internet fizeram evoluir as considerações e características dos sites conjuntamente. Isso faz com que a rastreabilidade dessas características possa nos ajudar a compreender melhor as novas formas de socialização entres pessoas ao redor da gastronomia P2P e considerar aquelas que possam ser trazidas a novos modelos de negócio gastronômico. Isto posto, os novos estilos de vida colaborativo são uma realidade cada vez mais presente, que deve ser considerada pelos agentes inseridos na atividade gastronômica. Os comportamentos de um público cada vez mais influenciado pelos conteúdos e formas de comunicação das redes sociais digitais, assim como o estudo das formas de socialização das pessoas a partir das próprias funcionalidades das plataformas de comunicação e seu retorno aos próprios negócios, são pesquisas interessantes para realizar no futuro. Também seria interessante, em futuros estudos, aprofundar-se sobre as tipologias das funcionalidades das caraterísticas aqui levantadas, segundo a atividade final que deseja fazer o usuário por meio das plataformas de compartilhamento de refeições. Posto que, nos objetivos da atividade final do usuário, há uma dicotomia: alguns focam na experiência de conhecer pessoas e novas culturas por meio de moradores locais usando a gastronomia como canal de socialização, enquanto outros priorizam a experiência gastronômica propriamente dita e preferem os serviços de um chef. Assim, a pesquisa sobre a ligação entre as funcionalidades das características das plataformas de compartilhamento de refeições e os objetivos da experiência dos usuários finais podem nos dar bons pontos de partida para futuras investigações.

\section{Referências}

Bauwens, M. (2005). The political economy of peer production. CTheory, 1.

Borges, R. M., Pinto, S. C. C. S., Barbosa, J. L., \& Barbosa, D. N. (2007). Usando o modelo 3 C de colaboração e Vygotsky no ensino de programação distribuída em pares. XVIII Simpósio Brasileiro de Informática na Educação (SBIE), 12-22.

Borghoff, U. M., \& Schlichter, J. H. (2000). Computer-supported cooperative work: Introduction to distributed applications. New York: Springer.

Botsman, R., \& Rogers, R. (2011). O que é meu é seu: Como o consumo colaborativo vai mudar o nosso mundo. Porto Alegre: Bookman.

De Oliveira, L. S., \& Gerosa, M. A. (2011). Collaborative features in content sharing Web 2.0 social networks: A domain engineering based on the $3 c$ collaboration model. In Collaboration and Technology (pp. 142157). Springer Berlin Heidelberg. 
Douglas, M., Isherwood, B., \& Dentzien, P. (2006). O mundo dos bens: para uma antropologia do consumo. Rio de Janeiro: UFRJ.

Douglas, M. (2007). O mundo dos bens, vinte anos depois. Horizontes Antropológicos, 13(28), 17-32.

Ellis, C.A., Gibbs, S.J. \& Rein, G.L. (1991) Groupware: Some issues and experiences. Communications of the ACM, 34(1), 38-58.

Foucault, M. (2004). Uma estética da existência. Ditos e escritos V: Ética, sexualidade, política. Rio de Janeiro: Forense Universitária.

Fragoso, S., Recuero, R., \& Amaral, A. (2011). Métodos de pesquisa para internet. Porto Alegre: Sulina.

Fuks, H. (2011). Sistemas colaborativos. Rio de Janeiro: Elsevier Brasil.

Gerosa, M. A., Raposo, A. B., Fuks, H., \& Lucena, C. J. P. (2006). Component-Based Groupware Development Based on the 3C Collaboration Model. Anais do XX Simpósio Brasileiro de Engenharia de Software-SBES, 129-144.

Gil, A. C. (2002). Como elaborar projetos de pesquisa. São Paulo: Atlas.

Kang, K. C. Et Al. (1990). Feature-Oriented domain analysis (FODA) feasibility study. Pittsburgh, PA: Software Engineering Institute.

Merino, M (2014). Cifras concretas sobre la repercusión de la economía colaborativa. Recuperado de www.pymes.ticbeat.com.

Michalsky, S., Mamani, E. Z. S., \& Gerosa, M. A. (2010). A inteligência coletiva na Web: Uma análise de domínio para o jornalismo online. Simpósio Brasileiro de Sistemas Multimídia e Web-Media, 2, 45-48.

Michalsky, S., \& Gerosa, M. A. (2010). Um modelo para o suporte computacional da inteligência coletiva na Web. Simpósio Brasileiro de Sistemas Colaborativos.

Oliveira, L. S. \& Gerosa, M. A. (2010). Uma engenharia de domínio baseada em padrões de interação e no modelo 3C de colaboração para redes sociais de compartilhamento na Web 2.0. Simpósio Brasileiro de Sistemas Multimídia e Web-Media, 2, 49-52.

Oliveira, L. S.. (2012). Interação em sistemas colaborativos. In H. Fuks (Org.). Sistemas colaborativos. Rio de Janeiro: Elsevier Brasil.

Pimentel, M., Gerosa, M. A., Filippo, D., Raposo, A., Fuks, H., \& Lucena, C. J. P. (2006). Modelo $3 C$ de Colaboração para o desenvolvimento de Sistemas Colaborativos. Anais do III Simpósio Brasileiro de Sistemas Colaborativos, 58-67.

Prieto-Diaz, R.; Arango, G. (1991). Domain analysis concepts and research directions. In: Prieto-Diaz, R., Arango, G. (Eds), Domain Analysis and Software Systems Modeling. Washington: IEEE Computer Society Press.

Rajanen, M.; livari,N. (2007) Usability cost-benefit analysis: how usability become a curve Word? Procceding of the 11 th IFIP TC 13 international conference on Human-computer interaction - Volume Part II. Rio de Janeiro, Brazil: Springer-Verlag.

Ramos; Brena. (2006). ¿Qué es la economía informal?. Número 60 del Observatorio de Economía Latinoamericana. Recuperado de http://www.eumed.net/cursecon/ecolat/index.htm

Romero A. (2014) Nuevos modelos de negocio en el sector turístico: implicaciones del escenario p2p. Congreso de AECIT: Asociación de Expertos Científicos en Turismo. Benidorm. 
Romero A.(2014). Potencial de los nuevos modelos de economía colaborativa en relación al turista. Recuperado de http://www.tecnohotelnews.com/2014/12/potencial-de-los-nuevos-modelos-de-economiacolaborativa-en-relacion-al-turista/

Rifkin, J. (2001). A era do acesso: A transição de mercados convencionais para networks e o nascimento de uma nova economia. São Paulo: Makron Books.

Tapscott, D., \& Williams, A. D. (2008). Wikinomics: How mass collaboration changes everything. London: Penguin.

Sharp, H., Rogers, Y., \& Preece, J. (2005). Design de interação: Além da interação homem-computador. Porto Alegre: Artmed.

Schummer, T.; Lukosch, S. (2007). Patterns for computer-mediated interaction. West Sussex: John Wiley \& Sons Ltd. 